\title{
Preface
}

\section{Towards practical applications in ensemble hydro-meteorological forecasting}

\author{
Y. He ${ }^{1, *}$, F. Pappenberger ${ }^{2}$, J. Thielen-del Pozo ${ }^{3}$, A. Weerts ${ }^{4}$, M.-H. Ramos ${ }^{5}$, and M. Bruen ${ }^{6}$ \\ ${ }^{1}$ Department of Geography, King's College London, Strand, WC2R 2LS, London, UK \\ ${ }^{2}$ European Centre for Medium-Range Weather Forecasts, Shinfield Park, Reading, RG2 9AX, UK \\ ${ }^{3}$ EC Joint Research Centre, IES, via E. Fermi, 2749, 21027 ISPRA (VA), Italy \\ ${ }^{4}$ Deltares, Inland Water Systems - Operational Water Management, Rotterdamseweg 185, P.O. Box 177, 2600 MH Delft, \\ The Netherlands \\ ${ }^{5}$ Cemagref, UR HBAN, 1, rue Pierre-Gilles de Gennes CS 10030, 92761 Antony Cedex, France \\ ${ }^{6}$ UCD School of Civil, Structural and Environmental Engineering, University College Dublin, Newstead, Belfield, \\ Dublin 4, Ireland \\ * now at: Tyndall Centre for Climate Change Research, School of Environmental Sciences, University of East Anglia, NR4 \\ 7TJ, Norwich, UK
}

We end the first decade of the 21 st century with memories of searing, vivid imagery of devastation, havoc and pain brought about by extreme hydro-climatic events. Of all these events, flooding particularly stands out. Pictures of deluges of unprecedented proportions in Australia, South America, Pakistan, West Africa and China, just to mention a few, demonstrate the increasing vulnerability of society and an eerie helplessness whenever our cities, towns and farmland are confronted by extreme floods. As the severity of floods seem to have increased in recent years, possibly due to both climate and landuse change, the need to invest more resources, financial and otherwise in hydro-meteorological forecasting as part of a multi-faceted approach to improving preparedness could not be greater. Engineers and scientists are increasingly under pressure to improve, not only the predictive capabilities of existing forecasting systems, but also the communication of forecast products to the right agencies for effective decision-making. Increasingly, decision makers seek information and the reliability and uncertainty in such forecasts. Ensemble forecasting techniques present an opportunity to enhance the value of current prediction methods especially when deliberate efforts are made at ensuring effective communication and use in decision-making.

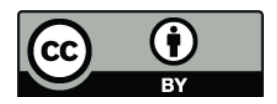

Correspondence to: $\mathrm{Y} . \mathrm{He}$

(yi.he@uea.ac.uk)
This special volume of Advances in Geosciences "Towards practical applications in ensemble hydrometeorological forecasting" solicited and received a large number of high quality submissions on the use of ensemble hydro-meteorological forecasting in a broad range of practical applications. A total number of 14 contributions are presented in this volume, covering the following areas:

1. Hydro-meteorological ensemble forecasting studies with different hydrological and climatic regimes that can lead to a reliable assessment of ensemble techniques;

2. Methods or standards developed to assess or benchmark the performance of ensemble rainfall-runoff modelling;

3. Pre- and post-processing of ensemble forecasts; and

4. Practical applications of ensembles in operational systems.

The contributions describe both the methodological and practical aspects of the topic, for instance, Randrianasolo et al. (2011) investigate the use of information from gauged neighbouring catchments to run a hydrological forecasting system in ungauged catchments and show that neighbouring catchments can help provide good quality forecasts at ungauged sites, especially when parameter sets are transferred for model simulation. In Alfieri et al. (2011a), a staggered approach to flash flood forecasting is developed within the

Published by Copernicus Publications on behalf of the European Geosciences Union. 
IMPRINTS project (FP7-ENV-2008-1-226555). They used the flash flood event that occurred on 2 November 2008 in the Cevennes region in France as a case study. Their description of the approach is complemented by Alfieri et al. (2011b), who describe a warning system based on rainfall threshold exceedances and discuss its results for a high-flow event in Catalonia, Eastern Spain. Wetterhall et al. (2011) compare precipitation data aggregated from hourly stations and data disaggregated from daily stations with 6-hourly forecasts from ECMWF over the time period October 2006 to end of 2009 and demonstrate that using sub-daily precipitation in the calibration and initiation of hydrological models can improve flood forecasting. Pappenberger et al. (2011) use a forecast consistency score (FCS) and analyse the system properties of a hydro-meteorological forecasting system (the European Flood Alert System) with respect to a fixed event. This paper adds an important perspective to the suite of forecast verification tools commonly used in this field. Based on 29 catchments in France, Velázquez et al. (2011) compare 16 different lumped hydrological model structures driven by weather forecasts from the European centre for medium-range weather forecasts (ECMWF) and evaluate the uncertainties associated with model structures and determine if performance improves when multiple hydro-models are implemented. Exbrayat et al. (2011) study the geographical transferability of different parameter sets and data-fusion methods applied to 5 different rainfall-runoff models for a catchment in Central Sweden. L. Zhao et al. (2011) evaluate the performance of a statistical post-processor for imperfect hydrologic model forecasts. The paper employs a "General Linear Model (GLM)" to post-process simulations produced by a hydrologic model. They show that the approach removes the mean bias effectively when applied to hydrologic model simulations from both the calibration and verification periods. Morawietz et al. (2011) study the performance of forecast post-processors that model any autocorrelation structure in forecast errors to improve the forecast. They found large differences in reliability and concluded that further work is required. Bao et al. (2011) study the potential benefits for early flood warning provided by a coupled atmospheric-hydrologic flood forecast model driven by the TIGGE ensemble forecasts for a catchment located in the upper reach of the Huai River basin in China. Schellekens et al. (2011) evaluate the performance of the MOGREPS Met Office forecast within the Environment Agency's (UK) National Flood Forecasting System (NFFS) for England and Wales. They demonstrate that such an ensemble system can provide better information to the forecaster than deterministic forecasts alone. In addition, they conclude that, with careful configuration in NFFS, MOGREPS can be used in existing systems without a significant increase in system load. The benefit of choosing ensemble forecasts over deterministic forecasts, when ensemble forecasts are corrected for resolution and bias with a simple post-processing technique, is shown in Boucher et al. (2011) for a flood event observed in the Gatineau River basin (Canada), influenced by reservoir operation constraints. L.-N. Zhao et al. (2011) compare the performance of the EPS produced by ECMWF, NCEP, CMA, and two multi-models with observations for the Chinese Huai River basin. They demonstrate that a Multi Model System (MMS) can outperform the individual forecasts of CMA and NCEP for most of the forecast days, although it is not as good as the ECMWF forecast. Ben Daoud et al. (2011) present an extended analog method to produce quantitative precipitation forecasts (QPFs). The method takes seasonal effects into account and uses information on the vertical velocity in the low troposphere $(850 \mathrm{hPa})$. It is designed to provide operational forecasts, in particular for hydroelectricity power production, for a large basin.

The overarching aim of the articles published in this special volume is to contribute to reducing the human suffering and material costs associated with floods, by addressing the issues related to the use of ensemble prediction systems and improved forecasting techniques. This ambition however remains constrained by shortcomings in the science, as well as management and institutional arrangements currently in place to identify, predict and manage floods. The volume is by no means conclusive and we hope it will rather open the door to a vibrant debate aimed at engaging a wider and more diverse audience.

\section{References}

Alfieri, L., Smith, P. J., Thielen-del Pozo, J., and Beven, K. J.: A staggered approach to flash flood forecasting - case study in the Cévennes region, Adv. Geosci., 29, 13-20, doi:10.5194/adgeo29-13-2011, 2011a.

Alfieri, L., Velasco, D., and Thielen, J.: Flash flood detection through a multi-stage probabilistic warning system for heavy precipitation events, Adv. Geosci., 29, 69-75, doi:10.5194/adgeo-29-69-2011, 2011b.

Bao, H.-J., Zhao, L.-N., He, Y., Li, Z.-J., Wetterhall, F., Cloke, H. L., Pappenberger, F., and Manful, D.: Coupling ensemble weather predictions based on TIGGE database with GridXinanjiang model for flood forecast, Adv. Geosci., 29, 61-67, doi:10.5194/adgeo-29-61-2011, 2011.

Boucher, M.-A., Anctil, F., Perreault, L., and Tremblay, D.: A comparison between ensemble and deterministic hydrological forecasts in an operational context, Adv. Geosci., 29, 85-94, doi:10.5194/adgeo-29-85-2011, 2011.

Ben Daoud, A., Sauquet, E., Lang, M., Bontron, G., and Obled, C.: Precipitation forecasting through an analog sorting technique: a comparative study, Adv. Geosci., 29, 103-107, doi:10.5194/adgeo-29-103-2011, 2011.

Exbrayat, J.-F., Viney, N. R., Seibert, J., Frede, H.-G., and Breuer, L.: Multi-model data fusion as a tool for PUB: example in a Swedish mesoscale catchment, Adv. Geosci., 29, 43-50, doi:10.5194/adgeo-29-43-2011, 2011.

Morawietz, M., Xu, C.-Y., and Gottschalk, L.: Reliability of autoregressive error models as post-processors for 
probabilistic streamflow forecasts, Adv. Geosci., 29, 109-118, doi:10.5194/adgeo-29-109-2011, 2011.

Pappenberger, F., Bogner, K., Wetterhall, F., He, Y., Cloke, H. L., and Thielen, J.: Forecast convergence score: a forecaster's approach to analysing hydro-meteorological forecast systems, Adv. Geosci., 29, 27-32, doi:10.5194/adgeo-29-27-2011, 2011.

Randrianasolo, A., Ramos, M. H., and Andréassian, V.: Hydrological ensemble forecasting at ungauged basins: using neighbour catchments for model setup and updating, Adv. Geosci., 29, 111, doi:10.5194/adgeo-29-1-2011, 2011.

Schellekens, J., Weerts, A. H., Moore, R. J., Pierce, C. E., and Hildon, S.: The use of MOGREPS ensemble rainfall forecasts in operational flood forecasting systems across England and Wales, Adv. Geosci., 29, 77-84, doi:10.5194/adgeo-29-77-2011, 2011.
Velázquez, J. A., Anctil, F., Ramos, M. H., and Perrin, C.: Can a multi-model approach improve hydrological ensemble forecasting? A study on 29 French catchments using 16 hydrological model structures, Adv. Geosci., 29, 33-42, doi:10.5194/adgeo29-33-2011, 2011.

Wetterhall, F., He, Y., Cloke, H., and Pappenberger, F.: Effects of temporal resolution of input precipitation on the performance of hydrological forecasting, Adv. Geosci., 29, 21-25, doi:10.5194/adgeo-29-21-2011, 2011.

Zhao, L., Duan, Q., Schaake, J., Ye, A., and Xia, J.: A hydrologic post-processor for ensemble streamflow predictions, Adv. Geosci., 29, 51-59, doi:10.5194/adgeo-29-51-2011, 2011.

Zhao, L.-N., Tian, F.-Y., Wu, H., Qi, D., Di, J.-Y., and Wang, Z.: Verification and comparison of probabilistic precipitation forecasts using the TIGGE data in the upriver of Huaihe Basin, Adv. Geosci., 29, 95-102, doi:10.5194/adgeo-29-95-2011, 2011. 